\title{
Phase Contrast Subtomogram Averaging at Sub-nm Resolution
}

Maryam Khoshouei ${ }^{1}$, Stefan Pfeffer ${ }^{1}$, Radostin Danev ${ }^{1}$, Friedrich Förster ${ }^{1}$, Wolfgang Baumeister ${ }^{1}$.

1. Department of Molecular Structural Biology, Max-Planck Institute for Biochemistry, Am Klopferspitz 18, , Martinsried, Germany.

Phase contrast imaging is a powerful technique that can increase the signal to noise ratio and contrast of the phase objects in their native and frozen hydrated state. The improved contrast is as a result of the phase shift between the unscattered and the scattered electron beams. The so called Volta phase plate (VPP) is a newly developed thin film phase plate which has advantages compared to the Zernike phase plate such as ease of use and the longer life time [1]. VPP is located in the back focal plane of the objective lens and produces phase shift based on the creation of beam-induced Volta potential above the continuous amorphous carbon film. In the case of the VPP, the phase shift is not constant and increases with the number of images while the Zernike phase plate has a phase shift of $90^{\circ}$. Recently, the VPP was used in the field of cryo-electron tomography and single particle analysis [2, 3]. VPP cryo-electron tomography has the potential to visualize molecular complexes in the intact cells [3].

Subtomogram averaging is a well-established method for structural visualization of macromolecular complexes by extracting the location and orientation of a specific complex from tomographic data. By tilting the sample through a range of angles (typically $-60^{\circ}$ to $+60^{\circ}$ ) and taking projection images, many copies of the same structural unit can be aligned and averaged to get a higher resolution structure $[4,5]$. One of the challenges in tomographic reconstruction is aligning different projections within the tilt series which can be improved by using the VPP. Additionally, the low frequency information is well and continuously transferred to the image, which is another strength of the phase contrast method compared to defocused based cryo-electron tomography (CTEM).

In this work, ice-embedded in vitro mammalian $80 \mathrm{~S}$ ribosomes were used to evaluate and compare the subtomogram averaging performance of the VPP and the conventional defocus method. Some structural details are more obvious in the VPP dataset such as expansion segments (Figure 1, red arrows). This suggests a benefit of using the VPP for more precise analysis of heterogeneous specimens which could be difficult to study by CTEM. From a fewer number of VPP subtomograms, higher resolution structure was obtained indicating that the VPP could be a helpful tool for achieving sub-nm resolution by subtomogram averaging. From CTF correction perspective in the data analysis, less processing is required because the data is collected close to focus. Furthermore, our results suggest that being close to focus and using no defocus offset during focusing are two critical parameters for achieving sub-nm resolution.

\section{References:}

[1] R Danev et al, Proceedings of the National Academy of Sciences of the United States of America 111 (2014), 15635-15640.

[2] S Asano et al, Science 347 (2015), 439-442.

[3] M Khoshouei et al, Nature Communications, doi: 10.1038/ncomms10534.

[4] F Forster and R Hegerl, Methods in Cell Biology 79 (2007), 741-767. 
[5] J.A Briggs, Current Opinion in Structural Biologycience 23 (2013), 261-267.
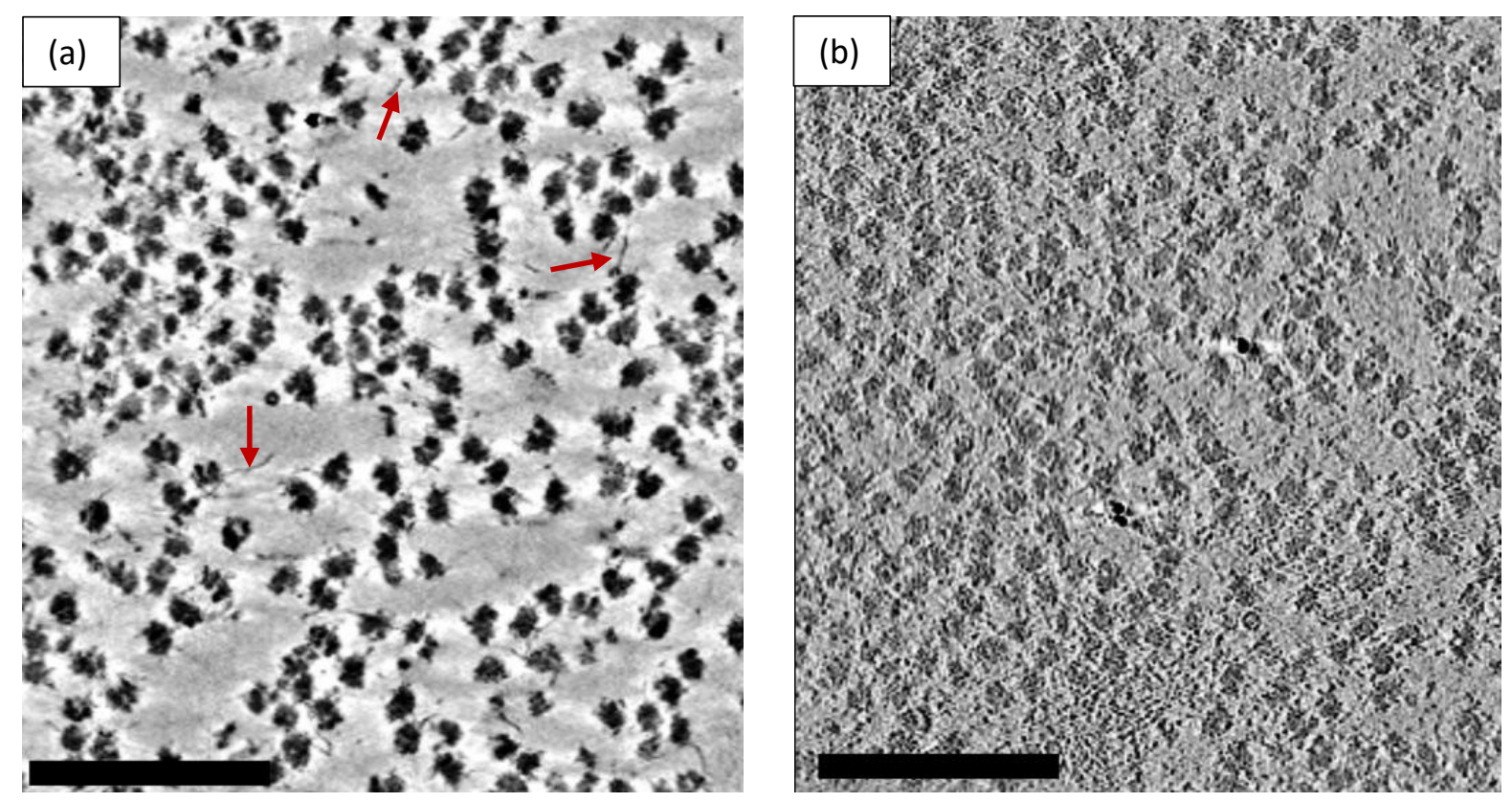

Figure 1. VPP vs CTEM cryo-electron tomography of mammalian $80 \mathrm{~S}$ ribosomes. (a) A slice from a single axis tilt VPP tomogram, defocus $-67.5 \mathrm{~nm}$. (b) A slice from a single axis tilt CTEM tomogram, defocus: $-3.5 \mu \mathrm{m}$. Scale bars: $200 \mathrm{~nm}$. Experimental conditions: Titan Krios 300kV, zero-loss energy filtering, K2 Summit direct detector, Magnification: 53000; Pixel size: $2.62 \AA$. 